\title{
The stomatognathic system in the elderly. Useful information for the medical practitioner
}

\author{
Anastassia E Kossioni' \\ Anastasios S Dontas ${ }^{2}$ \\ 'Department of Prosthodontics, \\ Dental School, University of Athens, \\ Greece; ${ }^{2}$ Hellenic Association of \\ Gerontology and Geriatrics, Athens, \\ Greece
}

\begin{abstract}
Aging per se has a small effect on oral tissues and functions, and most changes are secondary to extrinsic factors. The most common oral diseases in the elderly are increased tooth loss due to periodontal disease and dental caries, and oral precancer/cancer. There are many general, medical and socioeconomic factors related to dental disease (ie, disease, medications, cost, educational background, social class). Retaining less than 20 teeth is related to chewing difficulties. Tooth loss and the associated reduced masticatory performance lead to a diet poor in fibers, rich in saturated fat and cholesterols, related to cardiovascular disease, stroke, and gastrointestinal cancer. The presence of occlusal tooth contacts is also important for swallowing. Xerostomia is common in the elderly, causing pain and discomfort, and is usually related to disease and medication. Oral health parameters (ie, periodontal disease, tooth loss, poor oral hygiene) have also been related to cardiovascular disease, diabetes, bacterial pneumonia, and increased mortality, but the results are not yet conclusive, because of the many confounding factors. Oral health affects quality of life of the elderly, because of its impact on eating, comfort, appearance and socializing. On the other hand, impaired general condition deteriorates oral condition. It is therefore important for the medical practitioner to exchange information and cooperate with a dentist in order to improve patient care.
\end{abstract}

Keywords: stomatognathic system, elderly, oral disease, general health, xerostomia

\section{Introduction}

Many functions that are essential and sometimes characteristic to humans are performed by the stomatognathic system; speaking, chewing, tasting, swallowing, laughing, smiling, kissing, and socializing.

Ageing affects oral tissues, as any other part of the human body. However, many age-related changes apparent in mouth and in the functions of the stomatognathic system are secondary to extrinsic factors, other than age per se.

According to the Policy basis for the WHO Oral Health Programme, (a) oral health is integral and essential to general health, (b) it is a determinant factor for quality of life, (c) oral health and general health are interrelated, and (d) proper oral health care reduces premature mortality (Petersen 2003).

Findings in the mouth inform the physician about conditions that might affect the patient's overall health. On the other hand, systemic diseases and medications can cause oral diseases. Therefore, exchange of information between the physician and the dentist is very important. Moreover, impaired oral function is so common in the elderly that many physicians think that it can not be improved. However, there role to encourage their patients to seek dental help is valuable.

During the last 15-years Gerodontology has been included in the curriculae of many Dental Schools (Nitschke et al 2001; Mohammad et al 2003; Kossioni and Karkazis 2006). Three factors are mainly responsible: (a) the increased number of older persons 
in the present society, (b) their often complicated general condition that affects dental management, and (c) the changes in the dental disease and needs.

The aim of this paper is to describe the age-related changes in the stomatognathic system, to differentiate between the true age-changes and those related to other factors, to discuss the existing evidence about the interrelation of general health and oral health, and to identify ways to reduce their effect on the daily life of the elderly.

\section{The aging stomatognathic system The most common oral diseases}

The most common problems in the aging mouth are increased tooth loss (Figure 1), periodontal disease, dental caries (Figure 2) and oral precancer/cancer (Petersen 2003; Petersen and Yamamoto 2005). Tooth loss is related to periodontal disease, dental caries, impaired general medical condition and socioeconomic factors such as "dental fear", negative attitude towards oral health, increased cost, reduced access to dental services, low educational background, and low social class. The prevalence of edentulousness in the elderly reported by different studies in the various societies varies $(6 \%-78 \%)$ (Petersen 2003). However in many industrialized countries there is a trend towards decline in tooth loss (Pedersen and Yamamoto 2005).

Tooth loss is not inevitable as we grow old. Regular visits to the dentist and maintenance of proper oral care can reduce edentulousness.

\section{Motor performance}

Age changes in oral motor performance are not as evident as in other parts of the body (Baum et al 1991; Kossioni and Karkazis 1995, 1998). For example, simple masseteric

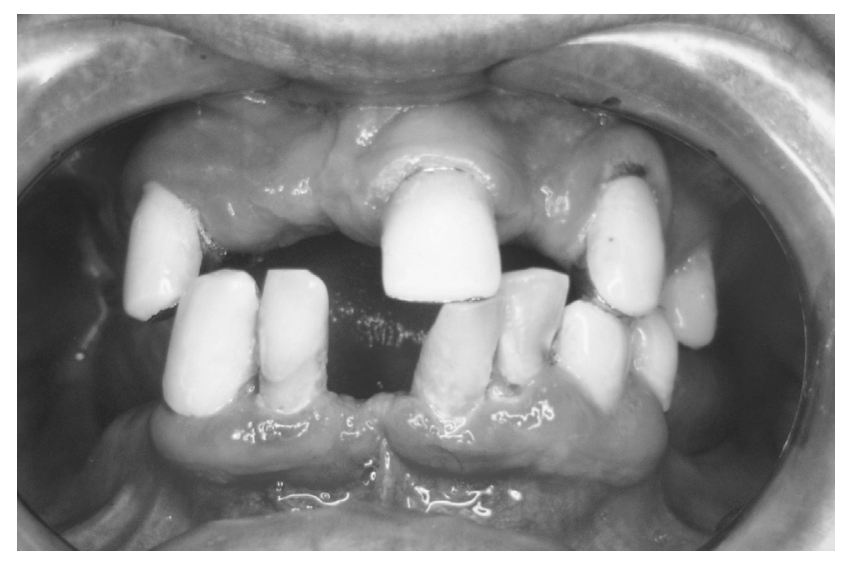

Figure I Extensive tooth loss is frequent in the elderly.

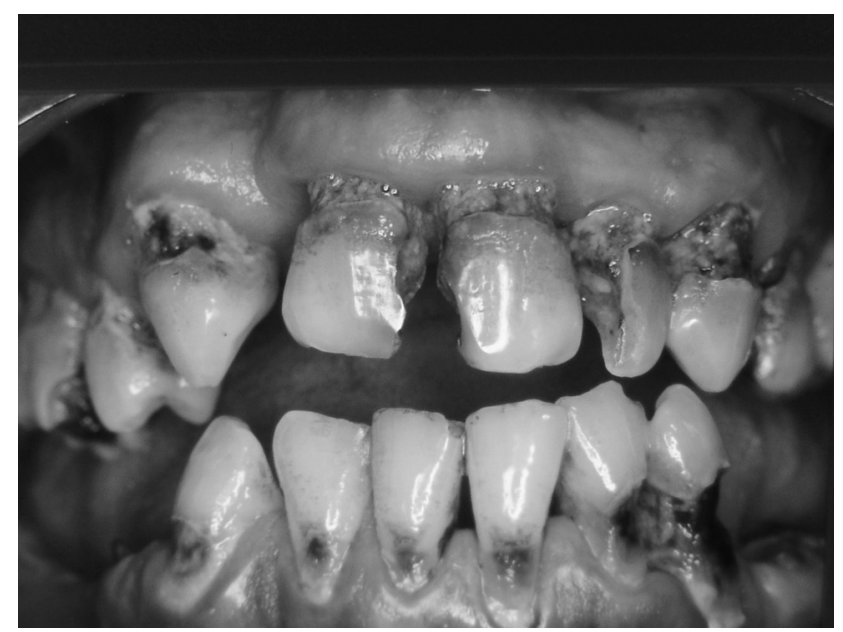

Figure 2 Root carries are common in the elderly.

reflex activity is maintained until very old age (Kossioni and Karkazis 1998). This is probably due to continuous use of jaw muscles for chewing, swallowing, speaking, smiling and to the particular functional role of these reflexes in humans.

Age per se has little effect on masticatory performance (Heath 1982; Carlsson 1984). Tooth loss is the main cause of reduced masticatory efficiency and affects food choice.

Although old people wearing dentures present similar patterns of functional adjustments to food consistency with dentate persons, indicating that the role of periodontal ligament mechanoreceptors is taken over by other receptors (Karkazis and Kossioni 1998), their masticatory performance differs in some respects. They have less masticatory strength (Heath 1982; Carlsson 1984), they need more time to chew the food (Helkimo et al 1978; Heath 1982), the movement velocity of the mandible is reduced (Jemt 1981) and their masticatory efficiency is reduced by $16 \%-50 \%$ (Heath 1982).

\section{Tooth loss, masticatory performance and diet}

Perceived masticatory ability increases with increasing numbers of natural teeth and/or pairs of opposing posterior teeth (Kossioni and Karkazis 1999; Sheiham et al 1999). People with more than 20 teeth present few chewing difficulties (Helkimo et al 1978; Witter et al 1990; Sheiham et al 1999).

People wearing full dentures, or people with extensive tooth loss consume less fruit, vegetables and meat and prefer food that is soft, rich in saturated fats and cholesterols (Laurin et al 1992; Johanson et al 1994; Krall et al 1998). This type of diet is associated with various medical conditions, ie, cardiovascular disease and gastrointestinal cancer. 
Moreover, compromised oral health (extensive tooth loss, absence of dentures) has been associated with nutritional deficiencies in very old, institutionalized and frail people, where reduced body mass index and serum albumin concentration have been recorded (Mojon et al 1999). In institutions, it is important to include a dentist and a dental hygienist in the care team (Mojon et al 1999).

People with dentures have specific difficulties eating hard consistency food (apples, raw carrots, meat), food with seeds which enter under the dentures causing discomfort and pain (tomatoes, grapes) and sticky foods attached to the acrylic dentures' teeth displacing the dentures (chocolate, gums) (Ettinger 1987). Denture problems also lead to social isolation, in order to avoid embarrassment from denture displacement, pain and discomfort during meals. Dental care can control those symptoms and improve quality of life of the edentulous persons (Figure 3).

However there are studies indicating that the correlation between masticatory variables and dietary selection is weak in the elderly (Osterberg et al 2002), while the improvement of dentures, or the fabrication of new ones have little effect on dietary selection (Sandstrom and Lindquist 1987; Laurin et al 1992). It is true that food selection is influenced by various factors such as cost, institutionalization, general medical condition, pain, xerostomia, loneliness, income, knowledge, culture, background etc (Heath 1982; Chen and Lowenstein 1984; Kossioni and Karkazis 1999; Sheiham et al 1999; Osterberg et al 2002). Chen and Lowenstein (1984) showed a positive

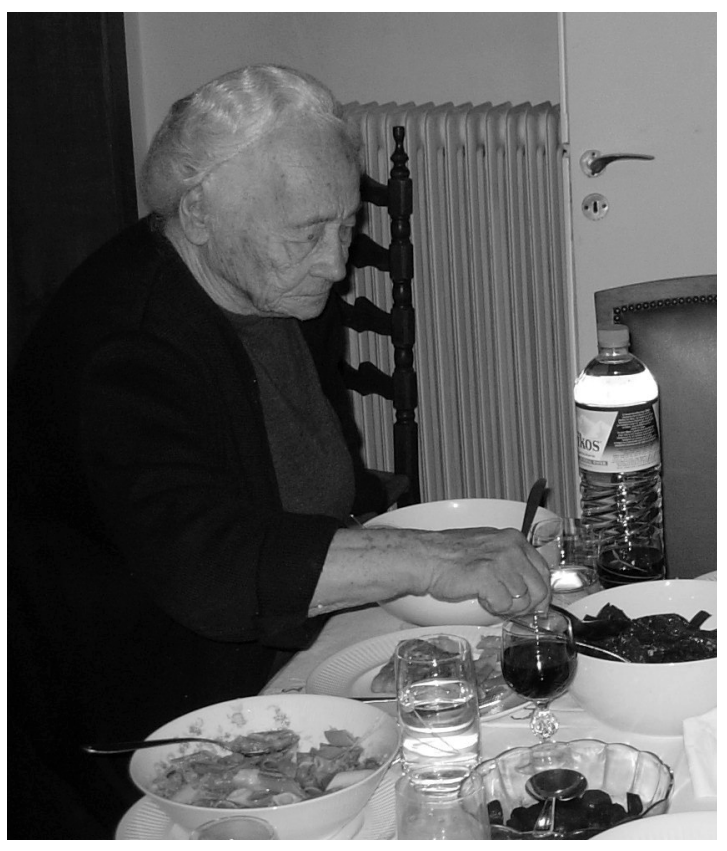

Figure 3 Chewing ability in the elderly is important to maintain a normal social life. correlation between masticatory problems and nutritional deficiencies and masticatory problems and hypertension, diabetes and ischemic heart disease only in people with a low socioeconomic background, indicating the role of general factors to food intake.

It is therefore very important to include dietary counseling, in order to improve dietary selection in people with compromised dentitions (Osterberg et al 2002).

\section{Xerostomia (oral dryness)}

Xerostomia is common in the elderly. Various studies report prevalence of subjective feelings of dry mouth of $12 \%-28 \%$, which increases to $40 \%-60 \%$ for those living in institutions (Narhi 1994; Ettinger 1996; Kossioni and Karkazis 1999; Field et al 2001). Dry mouth causes severe problems to the oral tissues and function, such as high incidence of caries, gingivitis, difficulties in chewing, swallowing, speaking and tasting, burning mouth, oral pain, oral malodour, increased vulnerability of the oral mucosa to mechanical trauma and microbial infection, and dentures' discomfort (Baum et al 1991; Haugen 1992; Narhi 1994; Sreebny and Schwartz 1997; Cassolato and Turnbull 2003; Petersen and Yamamoto 2005).

However, ageing is not the main cause of xerostomia. Xerostomia is usually related to systemic disease, medications and head and neck radiotherapy (Haugen 1992; Ettinger 1996; Sreebny and Schwartz 1997; Cassolato and Turnbull 2003). Many drugs are responsible including: antipsychotics (clozapine, chlorpromazine), tricyclic antidepressants (amitriptyline, imipramine, doxepin), selective serotonine reuptake inhibitors (sertraline, paroxetine, fluoxetine), antihypertensives (clonidine, methyldopa, prazosin), diuretics (spirnolactone, chlorothiazide, furosemide), sedatives (flurazapam, triazolam, temazepam) (Cassolato and Turnbull 2003). They mainly act by inhibiting signalling pathways within salivary tissue and reducing the output of the gland (Cassolato and Turnbull 2003).

It is very important to treat xerostomia because this will significantly improve the patients' quality of life. Treatment includes: regular review of drug regimens, reduction of total number of drugs, substitute xerostomic drugs with alternatives that have less xerostomic effect, modification of drug dose and schedule, intake of systemic cholinergic stimulants (pilocarpine HCL) if no contraindicated, frequent drinking of water and milk, use of saliva substitutes, use of saliva stimulants (chewing sugar free gum, sucking sugar-free lemon candies), avoiding smoking, avoiding dry food and alcohol consumption, frequent dental visits, improvement 
of denture fitting, improvement of oral hygiene, use of topical fluorides and antibacterial mouthwashes (chlorexidine 0.12\%) (Ettinger 1996; Sreebny and Schwartz 1997; Cassolato and Turnbull 2003).

A very close cooperation between the medical doctor and the dentist is imperative to control xerostomia and its side effects. Moreover, the people who are taking care of the frail institutionalized elderly must be informed about the problem and the necessary measures.

\section{Dysphagia (swallowing difficulty)}

Normal swallowing is very important to avoid aspiration pneumonia. It is little affected by age (Logemann 1990; Baum et al 1991), but it is affected by disease, xerostomia and unstable occlusion. The tongue and the mandible are stabilized in order to easily swallow hard food. This is best accomplished with the contraction of the jaw elevator muscles and the occlusion of the teeth (Zarb et al 1988). Therefore, providing occlusal stability by dental restorative treatment (ie, by fabricating bridges, partial dentures, and full dentures) helps swallowing function in the old age.

\section{Resorption of the alveolar bone}

After the extraction of the teeth the alveolar bone is gradually and continuously reducing (Klemetti 1996; Karkazis et al 1997; Carlsson 2004). In the study of Karkazis et al (1997) the overall reduction in the anterior mandibular height was $7.87 \mathrm{~mm}$ in a 7-years' period, but the interindividual variation was increased. The main clinical consequence of this problem is difficulty in the fabrication and functioning of complete dentures. Resorption is more pronounced in the mandible than in the maxilla and there are many old people who do not wear their lower dentures, as they find it difficult to control them.

The interindividual variation in bone loss is significant and the etiology is complex. Many factors have been implicated: ie, age, gender, length of edentulism, denture wearing habits, occlusal loading, nutrition, disease, medication, but the results are not conclusive (Klemetti 1996; Palmqvist et al 2003; Pietrokowski et al 2003; Carlsson 2004). Age itself has not yet proven to be of significant importance. Factors that seem to be involved are the female gender, the length of edentulism, pressure from denture wearing, and osteoporosis.

The best treatment of this problem is to prevent it by preserving natural teeth or even roots and construct appropriate prosthetic appliances (ie, overdentures). The placement of dental implants seems to provide functional stimulus to the bone and reduce bone loss (Carlsson 2004).

\section{Oral health and general health}

In the recent years there has been increased evidence of an interrelationship between oral and general health. This has been a subject of dispute for many years, and various studies have provided different results, because of the variations in study design, the population studied and the performed analysis (Barnett 2006). It is important to differentiate between results that indicate an association between the two conditions and those that prove a causal relationship (Barnett 2006). The main reason for this discussion is that both noncommunicable chronic diseases and oral diseases may have common risk factors related to lifestyle (eg, smoking, diet) (Petersen 2003; Demmer and Desvarieux 2006).

Oral manifestations of systemic diseases, if early detected, could be extremely beneficial to the patient in terms of diagnosis (eg, microbial infections, immune disorders, nutritional deficiencies) (Petersen 2003).

There is an increasing number of publications on the connection between periodontal disease and diabetes, oral infection and cardiovascular disease and oral infection and bacterial pneumonia. However the results are not clear and conclusive yet.

Inflammation is a common link between periodontal disease and diabetes, and there is some evidence indicating that diabetes is associated with an increased risk of developing periodontal disease, while the effect of periodontal disease on diabetes is less clear (Mealey 2006). Patients with diabetes and poor glycemic control have increased risk of developing periodontitis compared to patients with well-controlled diabetes, but periodontal treatment has variant effect on glycemic status (Mealey 2006).

Oral health parameters (eg, gingivitis involving bleeding, 1 to 14 natural teeth, xerostomia complaints, low salivary levels of S. Sanguis, positive plaque benzoyl-DL-argininenaphtylamide test scores) and coronary heart disease have been associated in a convenience sample of 320 US veterans who were at least 60-years of age, but the results can not be generalized (Loesche et al 1998). Regarding periodontal disease and cardiovascular disease there is evidence supporting a moderate relative epidemiological association, more pronounced in younger subjects and stronger for cerebrovascular disease than for coronary disease, but a causal effect is not yet proved (Demmer and Desvarieux 2006). However, although there is no scientific evidence that preventing or treating periodontal disease will reduce cardiovascular 
disease, periodontal treatment is recommended because of the general benefit to the patients (Demmer and Desvarieux 2006). A significant association has been recorded between tooth loss levels (as a marker of past periodontal disease) and carotid artery plaque prevalence (Desvarieux et al 2003). Periodontal disease and fewer teeth have also been associated to ischemic stroke, but further studies are needed to be conducted to clarify whether those are independent risk factors (Joshipura et al 2003). Incident tooth loss has also been associated with peripheral arterial disease, especially among men with periodontal disease (Hung et al 2003).

Institutionalized elderly with respiratory tract infections have significantly higher levels of dental plaque, containing bacteria that can cause chest infections (Department of Health 2005). Infections originate from dental plaque on unclean teeth and dentures. Poor oral hygiene and the oral microflora have been specifically related to nosocomial ventilator-associated bacterial pneumonia in special-care populations, but there is little evidence of an association between poor oral hygiene and periodontal disease and community acquired pneumonia (Scannapieco 2006). The presence of selected oral disorders (edentulous with defective denture bases or generalized stomatitis and dentate with visible calculus (Figure 4), generalized gingivitis, and teeth with pulpal exposures) associated with low serum albumin $(<35 \mathrm{~g} / \mathrm{L})$ increased the relative risk of respiratory tract infection to 3.2 (1.5-6.7), while it was 1.9 among subjects with oral problems and ALB $>35 \mathrm{~g} / \mathrm{L}$ (Mojon et al 1997). The incidence of respiratory tract infection in frail institutionalized elderly was greater among dentate subjects and those who consulted a dentist in emergency (Mojon et al 1997). Preventive dental care is important in preventing serious lung infections in institutional settings (Mojon et al 1997;

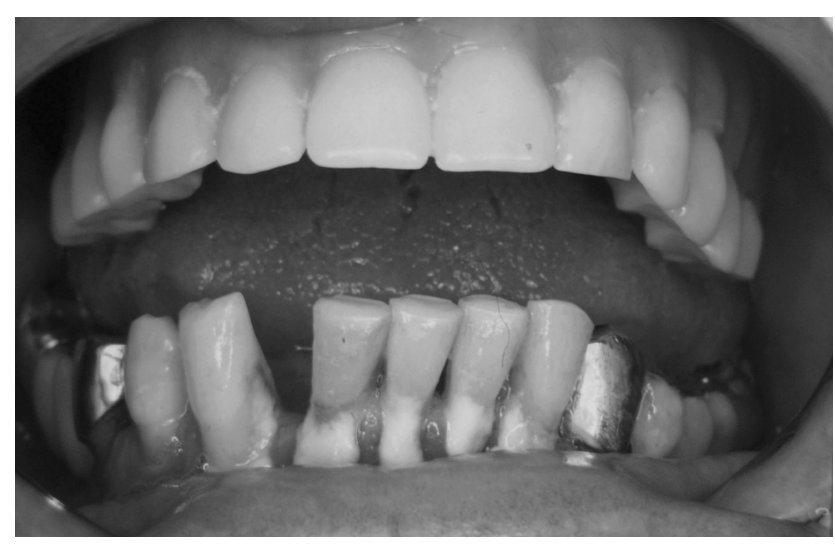

Figure $4 \mathrm{Visible}$ calculus on the labial surfaces of the lower teeth of an older female, due to poor oral hygiene.
Scanapieco 2006). However the most efficient method is not clear yet (use of topical oral disinfectant with chemotherapeutic agents alone or with mechanical oral hygiene methods) (Scannapieco 2006).

A multivariate analysis adjusted for general health and lifestyle factors in a cohort born in 1910 in Finland, mostly living at home, has shown that the risk for death of old subjects with urgent need of dental treatment was 3.9 times higher than that of other subjects (Hamalainen et al 2005). Urgent need of dental treatment was regarded as pain, infection or condition leading to serious consequences if not treated (ie, chronic periapical abscess, acute necrotizing ulcerative gingivitis, oral abscess, signs of oral cancer). The authors concluded that it is important to diagnose oral infections in time and eradicate them as soon as possible in order to prevent severe complications.

On the other hand, frail elderly often have deteriorated oral care because of impaired vision and manual dexterity, diminished muscle control and oral status susceptible to oral plaque accumulation (Walls and Steele 2001). There are also problems from systemic diseases and medications causing xerostomia, altered smell and taste, and various oral diseases (Kossioni and Karkazis 1999, Pedersen and Yamamoto 2005).

It is very important to include a dentist and a hygienist in the frail elderly care team and also to educate the nurses and the carers to perform oral hygiene for those who are unable to do it themselves.

\section{Oral health and quality of life}

Oral health affects quality of life (McGrath and Bedi 1999; Pedersen 2003; Pedersen and Yamamoto 2005).

Old people consider as important the impact of oral health especially on eating, comfort, carefree manner and appearance (McGrath and Bedi 1999). However, variation is recorded in the preferences between different sexes and social classes (McGrath and Bedi 1999). More men than women consider eating as important and more women confidence.

A very important factor is tooth loss. It has been related to difficulties in eating, pain, distress and social isolation (Jones et al 2003). Old people with more than 20 teeth present had better subjective physical health than those with fewer teeth (Akifusa et al 2005).

\section{Conclusion}

There are many changes in the stomatognathic system with aging that can affect quality of life (tooth loss, masticatory problems, xerostomia, dysphagia). Impaired oral health is 
common, mainly due to the rare visits to the dentist and the often neglected oral hygiene. There is also increased evidence of an association between oral disease and systemic disease, especially in the frail elderly. Thus, exchanging information between the physician and the dentist is important. Oral care (preventive, restorative and surgical) will benefit the elderly. The medical practitioner should pay attention to the oral problems of the older subjects and encourage them to seek dental care.

\section{References}

Akifusa S, Soh I, Ansai T, et al. 2005. Relationship of number of remaining teeth to health-related quality of life in community-dwelling elderly. Gerodontology, 22:91-7.

Barnett ML. 2006. The oral-systemic disease connection. JADA, 137:5S-6S

Baum BJ, Caruso AJ, Ship JA, et al. 1991. Oral physiology. In: Papas A, Chauncey $\mathrm{H}$ eds. Geriatric dentistry. Aging and oral health. St Louis, Mosby year Book, 71-82.

Carlsson GE. 1984. Masticatory efficiency: the effect of age, the loss of teeth and prosthetic rehabilitation. Int Dent $J, 34: 93-7$.

Carlsson GE. 2004. Responses of jaw bone to pressure. Gerodontology, 21:65-70.

Cassolato SF, Turnbull RS. 2003. Xerostomia: clinical aspects and treatment. Gerodontology, 20:64-77.

Chen MK, Lowenstein F. 1984. Masticatory handicap, socioeconomic status and chronic conditions among adults. JADA, 109:916-18.

Demmer RT, Desvarieux M. 2006. Periodontal infections and cardiovascular disease. The heart of the matter. JADA, 137:14S-20S

Department of Health. 2005. Meeting the challenges of oral health for older people: a strategic review 2005. Gerodontology, 22 (Suppl 1):9-11.

Desvarieux M, Demmer RT, Rundek T, et al. 2003. Relationship between periodontal disease, tooth loss, and carotid artery plaque. The oral infections and vascular disease epidemiology study (INVEST). Stroke, 34:2120-5.

Ettinger RL. 1987. Oral disease and its effect on the quality of life. Gerodontics, 3:103-6.

Ettinger RL. 1996. Xerostomia: a symptom which acts as a disease. Age and Ageing, 25:409-12.

Ettinger RL, Watkins C, Cowen H. 2000. Reflections on changes in geriatric dentistry. J Dent Educ, 64:715-722.

Field EA, Fear S, Higham SM, et al. 2001. Age and medication are significant risk factors for xerostomia in an English population, attending general dental practice. Gerodontology, 18:21-4.

Haugen LK. 1992. Biological and physiological changes in the ageing individual. Int Dent $J, 42: 339-48$.

Hamalainen P, Meurman J, Kauppinen M, et al. 2005. Oral infections as predictors of mortality. Gerodontology, 22:151-7.

Heath MR. 1982. The effect of maximum biting force and bone loss upon masticatory function and dietary selection of the elderly. Int Dent $J$, $32: 345-56$.

Helkimo E, Carlsson GE, Helkimo M. 1978. Chewing efficiency and state of dentition. Acta Odont Scand, 36:33-41.

Hung H-C, Willett W, Merchant A, et al. 2003. Oral health and peripheral arterial disease. Circulation, 107:1152-7.

Jemt T. 1981. Chewing patterns in dentate and complete denture wearers recorded my light-emitting diodes. Swed Dent J, 5:199-205.

Joshipura KJ, Hung H-C, Rimm EB, et al. 2003. Periodontal disease, tooth loss, and incidence of ischemic stroke. Stroke, 34:47-52.

Johansson I, Tidehag P, Lundeberg V, et al. 1994. Dental status, diet and cardiovascular risk factors in middle-aged people in northern Sweden. Community Dent Oral Epidemiol, 22:431-6.
Jones JA, Orner MB, Spiro III A, et al. 2003. Tooth loss and dentures: patients' perspectives. Int Dent J, 53:327-34.

Karkazis HC, Kossioni AE. 1993. Oral health status, treatment needs and demands of an elderly institutionalized population in Athens. Eur $J$ Prosthodont Rest Dent, 1:157-63.

Karkazis HC, Lambadakis J, Tsiklakis K. 1997. Cephalometric evaluation of the changes in mandibular symphysis after 7 years of denture wearing. Gerodontology, 14:101-5.

Karkazis HC, Kossioni AE. 1998. Surface EMG activity of the masseter muscle in denture wearers during chewing of hard and soft food. J Oral Rehab, 25:8-14.

Klemetti E. 1996. A review of residual ridge resorption and bone density. $J$ Prosthet Dent, 75:512-14.

Kossioni AE, Karkazis HC. 1995. Variation in the masseteric silent period in older dentate humans and in denture wearers. Archs Oral Biol, 40:1143-50

Kossioni AE, Karkazis C. 1998. Jaw reflexes in healthy old people. Age and Ageing, 27:689-95.

Kossioni AE, Karkazis HC. 1999. Socio-medical condition and oral functional status in an older institutionalized population. Gerodontology, 16:21-8.

Kossioni AE, Karkazis HC. 2006. Development of a gerodontology course in Athens: a pilot study. Eur J Dent Educ, 10:131-6.

Krall E, Hayes C, Garcia R. 1998. How dentition status and masticatory function affect nutrient intake. JADA, 129:1261-9.

Laurin D, Brodeur J-M, Leduc N, et al. 1992. Nutritional deficiencies and gastrointestinal disorders in the edentulous elderly: a literature review. $J$ Can Dent Assoc, 58:738-40.

Loesche WJ, Schork A, Terpenning MS, et al. 1998. Assessing the relationship between dental disease and coronary heart disease in elderly US veterans. JADA, 129:301-11.

Logemann JA. 1990. Effects of aging on the swallowing mechanism. Otolaryngol Clin North Am, 23:1045-56.

McCord JF, Wilson MC. 1994. Social problems in geriatric dentistry: an overview. Gerodontology, 11:63-6.

McGrath C, Bedi R. 1999. The importance of oral health to older people's quality of life. Gerodontology, 58-63.

Mealey BL. 2006. Periodontal disease and diabetes. A two-way street. $J A D A, 137: 26 \mathrm{~S}-31 \mathrm{~S}$

Mohammad AR, Preshaw PM, Ettinger RL. 2003. Current status of predoctoral geriatric education in US dental schools. J Dent Educ, 67:509-14.

Mojon P, Budtz-Jorgensen E, Michel J-P, et al. 1997. Oral health and history of respiratory tract infection in frail institutionalised elders. Gerodontology, 14:9-16.

Mojon P, Budtz-Jorgensen E, Rapin C. 1999. Relationship between oral health and nutrition in very old people. Age and Ageing, 28:463-8.

Narhi TO. 1994. Prevalence of subjective feelings of dry mouth in the elderly. J Dent Res, 73:20-5.

Nitschke I, Muller F, Ilgner A, et al. 2001. Undergraduate teaching in gerodontology in Austria, Switzerland and Germany. Gerodontology, 21:123-9.

Osterberg T, Tsuga K, Rothenberg E, et al. 2002. Masticatory ability in 80 -year-old subjects and its relation to intake of energy, nutrients and food items. Gerodontology, 19:95-101.

Palmqvist S, Carlsson GE, Owall B, et al. 2003. The combination syndrome: a literature review. J Prosthet Dent, 90:270-5.

Petersen PE, Yamamoto T. 2005. Improving the oral health of older people: the approach of the WHO Global Oral Health Programme. Community Dent Oral Epidemiol, 33:81-92.

Petersen PE. 2003. The world oral health report 2003: continuous improvement of oral health in the 21 st century- the approach of the WHO global oral health programme. Community Dent Oral Epidemiol, 31(Suppl 1):3-24.

Pietrokowski J, Harfin J, Levy F. 2003. The influence of age and denture wear on the size of edentulous structures. Gerodontology, 220:100-5.

Sandstrom B, Lindquist LW. 1987. The effect of different prosthetic restorations on the dietary selection in edentulous patients. Acta Odont Scand, 45:423-8. 
Scannapieco FA. 2006. Pneumonia in nonambulatory patients. The role of oral bacteria and oral hygiene. JADA, 137:21S-25S.

Sheiham A, Steele JG, Marcenes W, et al. 1999. The impact of oral health on stated ability to eat certain foods; findings from the National Diet and Nutrition Survey of Older people in Great Britain. Gerodontology, 16:11-20.

Sreebny LM, Schwartz SS. 1997. A reference guide to drugs and dry mouth2nd edition. Gerodontology, 14:33-47.

Walls AWG, Steele JG. 2001. Geriatric oral health issues in the United Kingdom. Int Dent J, 51:183-7.

Witter DJ, Cramwinckel AB, van Rossum GMJM, et al. 1990. Shortened dental arches and masticatory ability. J Dent, 18:185-9.

Zarb GA, Mohl ND, Mackay HF. 1988. Deglutition, respiration and speech. In: Mohl ND, Zarb GA, Carlsson GE eds. A textbook of occlusion. Chicago: Quintessence Publishing Co Inc. p 153-60. 
